\title{
The Semantic Understanding of the English Counterfactual Conditionals-A Model Based on Conceptual Integration Theory
}

\author{
Ling Qin \\ English Department, Sichuan Agricultural University, Ya'an, China
}

\begin{abstract}
This research refers to the previous studies on the semantic understanding of the English Counterfactual Conditionals linguistically and aims at revising the Conceptual Integration Theory to find out a better model of explaining the semantic understanding of the English Counterfactual Conditionals. Through the theoretical inference and analysis of some live samples of these conditionals, the validity and explanatory power of the new model are defined.
\end{abstract}

Index Terms—semantics, counterfactual conditional, CIT

\section{INTRODUCTION}

Counterfactual way of thinking is a common living way in which we human beings think and even start our intelligence. In English, counterfactual meanings are indicated by some specific verb patterns and grammatical structures. The pedagogic grammar rules provide the descriptions and regulations of how these meanings are organized in phrases and sentences. Inspiringly it is noticed that the counterfactual if-conditionals, which bear the typical and prominent structures in grammar. Anyway, from a cognitive perspective, Fauconnier (1997) language is a superficial manifestation of hidden, highly abstract, and cognitive constructions (p.34) English Counterfactual Conditionals ${ }^{1}$, as a kind of important structure of 'subjunctive mood', is a good sample of counterfactuality to study, under the guidance of relevant cognitive theories.

We can study the etymology of 'subjunctive' to find that 'subjunctive' means 'proper to be subjoined', which means that subordinates, mainly are the major manifestation of the counterfactual property. As a matter of fact, English subjunctive mood has left little forms in use, because of the vanishing of some inflexions in live present English text. ECC are the most commonly used form nowadays. Meanwhile, ECC research is commonly regarded as a grammatical structure analysis in some researches and has left some unsolved problems to deal with. However, critically, ECC is not simply the manifestation of some inflexions in grammatical structure, but also more importantly convey the cognitive psychology of the unreal in human way of thinking. That's to say, the study of ECC is necessary to be put to more research cognitively.

Dancygier \& Sweetser (2005) write that Conditionality or conditional reasoning is also important in human thought. "Philosophers have long focused on conditional constructions as manifestations of human logical reasoning... Psychologists, philosophers and anyone who studies human reasoning should be interested in the unique and persuasive cognitive patterns displayed in conditions." (p. 56) Conditionality is not only a philosophical concern, but also is a major concern in linguistics. The property of conditionality arouses linguistic insights of grammar, semantics and cognitive approaches.

In the selection of linguistic theories to apply to this research, Conceptual Integration Theory ${ }^{2}$ proposed by Fauconnier and Turner in a multitude of their studies and books can be thought to be proper in this research, because CI can better explain the backstage running of the dynamic meaning-form construction of ECC. Secondly, though some application researches have been done by adopting CI to the study of ECC, they have not yet provided a general model which can explain the general cognitive rules under which ECC' meaning is generated and is also comprehended.

ECC embodies the integrated property of counterfactuality and conditionality. Besides grammatical, semantic and pragmatic approaches, the cognitive mechanism of ECC needs to be looked into in a way where the accounts of counterfactuality and conditionality are taken organically together.

Based on this discussion, the present research is both necessary and practicable.

The research will apply CI to the theoretical analysis; the original theoretical aspects are reviewed and evaluated. To review them, the thesis will sort out the relative different scholarly viewpoints, mainly CI theory and its derivation. Accordingly, conditionality and its study will be reviewed.

Another but the most important objective of this research is to provide a pattern under the guidance of which common rules of how ECC meaning is indicated can be well defined. As we all know, CI has offered a description of

\footnotetext{
${ }^{1}$ shortened as ECC in this thesis, as is explained in 1

${ }^{2}$ Shortened as CI in this thesis
} 
how our brains work in the generation of semantics and the comprehension. In more detailed way, a framework must be constructed according to the generals of CI for the explanation of ECC. In this pattern, an integrated account will be taken to build spaces and network(s) to analyze ECC in live English text. In the application of the theories, the thesis will analyze the relative structures in English language and will explain further about the pattern proposed through the research.

In the use of the sample structures, the research will employ live English counterfactual conditionals, collected randomly as the corpora. The corpora are open; random sentences or texts valid in live English language are analyzed to testify the common explanatory capability of the pattern in this research. The classifications of these sentences will be done according to the pattern in the present thesis as the justification of the theoretical approach. CI has been applied to 'subjunctive mood' and some researchers have applied the theory in the study of ECC. The present research will reserve the valuable assertions.

The analyses of CI and its extended contents, along with the application of it to ECC examples, will discover how the meaning of ECC is generated and understood by people. A mode is given in the summary of the study that helps to explain the issue in general; this part is the aim and result of the research.

Limitations of the relevant theoretical approaches will be discussed, too, and the suggestions will be made for the future studies on the issue. The description of these limitations and suggestions will also be one of the important parts as the motivation of the present thesis.

As a qualitative theoretical study, this research collects ECC that are needed in the analysis. The published studies of ECC are introduced in the literature, whose focus has been put either on the cognitive mechanism of counterfactuality or on that of conditionality. CI applies to 'subjunctive mood' and some researchers have put the theory in the study of ECC. The present research reserves the valuable assertions.

The original CI is firstly adapted to the further analysis and a pattern is constructed to explain. Again, the pattern is testified in the analysis of randomly chosen ECC. In the application of the theory, conditionality and its study are considered too, as is proclaimed in the previous verse.

In the use of the sample structures, the research employs live English counterfactual conditionals, collected randomly as the corpora. The corpora are open, and some random sentences or texts valid in live English language are analyzed to testify the common explanatory capability of the pattern in this research. The classification of these sentences is specified according to pedagogic grammar and the sentences are analyzed respectively based on the pattern originated from $\mathrm{CI}$.

\section{Counterfactuality And Counterfactual Conditional}

In understanding and indicating the meaning of counterfactuality, a question must be asked in advance: how do human beings determine whether the meaning of a condition is true or false? David Lewis (1973) analyzed the truth conditions and the logic of counterfactuals in terms of possible world semantics. (p.94) The counterfactuality is considered as a statement about how things run in other possible worlds managed by the same laws of nature.

Besides logic-semantic perspective, counterfactuality is also viewed as complex, dynamic cognitive property. Fauconnier and Turner (2002) note as "counterfactual scenarios are assembled mentally not by taking full representations of the world and making discrete, finite, known changes to deliver full possible worlds but, instead, by conceptual integration, which can compose schematic blends that suit the conceptual purposes at hand."(p.218)

As in Oxford English Dictionary (1989), "COUNTERFACTUAL" is, as an adjective, Pertaining to, or expressing, what has not in fact happened, but might, could, or would, in different conditions; counterfactual conditional, a conditional statement of this sort, normally indicating its character by the use of the subjunctive mood in its protasis." (Vol. III: p. 1026) The Oxford English Dictionary definition of "subjunctive" is to "designating a mood, the forms of which are employed to denote an action or a state as conceived (and not as a fact) and therefore used to express a wish, command, exhortation, or a contingent, hypothetical, or prospective event" (Vol. XVII: pp. 35-36).

According to Quirk et al (1985), a hypothetical condition is also termed a "closed", "unreal", "nonfactual" or "counterfactual" condition. It "conveys the speaker's belief that the condition will not be fulfilled (for future conditions), is not fulfilled (for present conditions), or was not fulfilled (for past conditions)". (p.1097) They (Quirk et al, 1985) also provided a diagram to demonstrate the verb forms with "hypothetical (counterfactual, in this paper) conditions" (p.1088), as in Table 2.1:

TABLE 2.1:

VERB FORMS WITH HYPOTHETICAL CONDITIONS

\begin{tabular}{|l|ll|}
\hline & conditional clause & matrix clause \\
\hline $\begin{array}{l}\text { present and } \\
\text { future reference }\end{array}$ & PAST & PAST MODAL \\
\hline past & PAST PERFECTIVE & I would study Classical Greek. \\
reference & If I had seen you, & PAST PERFECTIVE MODAL \\
\hline
\end{tabular}


From the perspective of semantics, counterfactual conditional gains its own features. Discussed by Leech (1987), different verbal constructions in English indicate three different meanings_- "factual", "theoretical" and "hypothetical (counterfactual, in this paper)" (p.106). Fillmore (1990a, 1990b) set two parameters-“epistemic stance" and "tense structure", on which English conditionals analysis depends, for ECC require the verb form which has an interpretation of the protasis negative in epistemic stance, and the apodosis negative in epistemic stance, too. (pp. 49-56; pp. 57-76)

Grammatically, specific verb forms are taken to indicate definitely present or future counterfactual conditional. In present counterfactual conditional, "BE" is specified to "WERE" even if matched with the first-singular or the thirdsingular person to indicate the counterfactual meaning. In the future counterfactual subordinate, auxiliary "WERE TO" (or as a modal phrase) is used. However, these forms are used less frequently nowadays, especially in oral language, and Quirk et al (1985:158) called them "fossilized inflections".

The following description of specific verb forms of ECC is a comprehensive review of various grammarians' studies, such as Quirk et al's (1985), Zhang Daozhen's (2002), Zhang Zhenbang's (1984), Bo Bin et al's (1978) and Bo Bin's (1994) with the classification and assortment in this research.

From the perspective of time, verb forms vary according to the supposition contrary to the present-fact, to the pastfact and to the possible future in ECC. Besides, "mixed-time" happens when the subordinate and the matrix clause take different time.

The diagram is to illustrate in Table 2.2:

TABLE 2.2:

VERB FORMS IN ECC ${ }^{3}$

\begin{tabular}{|l|l|l|}
\hline Time & Verb Form in the Subordinate & Verb Form in the Matrix clause \\
\hline Present & $\begin{array}{l}{[\mathrm{DID}]} \\
\text { (be } \rightarrow \text { were })^{4}\end{array}$ & Would/should/could/might... ${ }^{5}[\mathrm{DO}]$ \\
\hline Future & $\begin{array}{l}{[\mathrm{DID}]} \\
\text { Were to [DO] }\end{array}$ & Would/should/could/might... [DO] \\
\hline Past & had [DONE] & Would/should/could/might...have [DONE] \\
\hline
\end{tabular}

We also notice that the truth-conditional subordinates, certain words and phrases, other kinds of subordinates such as, the attributive clause or the objective clause, can imply counterfactual conditions. Context is also utilized in this implication. In these forms, the subordinates (or the equal semantic structures) are not "counterfactual" or even "conditional", but the matrix clause, yet with specific forms, also indicates the counterfactual meaning. ${ }^{6}$

Examples of these structures are in Appendix I.

\section{STUDIES OF ECC}

Goodman (1947) began the modern researches on counterfactuality. According to his essay "The Problem of Counterfactual Conditionals", the following analysis of the counterfactual conditional" $p \square \rightarrow q$ "is read"If $p$ were true, then $q$ would be true." (p.58) $p \square \rightarrow q$ if and only if p nomologically requires, given prevailing conditions, the truth of $q$. Or, in other words, if $p$, conjoined with some set of facts $S$ and laws of nature $L$, deductively entails q. More formally, we have:

$$
\text { (C) } p \square \rightarrow q \text { if and only if }\{p, S, L\} \perp=q
$$

Suppose (as is typically the case) that $p$ and $q$ are (in fact) both false. If we were to include $\neg q$ in the "set of facts" $S$, then Goodman's $\{p, S, L\}$ would be unsatisfiable, and so $p \square \rightarrow q$ would be true, trivially. For example, we accept "If the war had been prevented, the civilians in battle-field would have survived" because the war is blood-shedding and people are killed in it. We do not, however, (pace Goodman) accept "If the war had been prevented, the war wouldn't have been blood-shedding" even though people are not in fact killed. Not wanting to make all (interesting) counterfactuals true, Goodman suggested that we constrain $S$ in such a way that it include only facts "that would not be altered by the truth of (i.e., those cotenable with) p." The problem with this response is that it constrains $S$ using, essentially, another counterfactual. In essence, Goodman's"fix"is to add the constraint that $p$ a $\rightarrow$. But, this is viciously circular.

To explain what "law" is, Goodman suggests a generalization of the form "All A's are B's" is a law if it is "projectible" - that is, if the observation of A's that are B provides reason to believe that unobserved A's are B. But, when does this happen? It seems that there are many non-lawlike generalizations that are (legitimately) "projected" from small samples. All it takes for a generalization to be projectible is the belief that its truth would not just be a coincidence, but there is some uniform reason for its conformity - they cannot be "wholly accidental".

\footnotetext{
${ }^{3}$ In mixed-time ECC, verb forms are taken according to what time it takes in their own clauses.

${ }^{4}$ [DID] is a parameter which means the past form of any verb including its passive voice or other proper inflexions, and any capitalization in the squared brackets "[ ]" means the similar. The structure "(be $\rightarrow$ were)" is the explanation which means in this situation, verb "be" should be transformed into "were".

${ }^{5}$ The symbolic structure " $/ . . . / . . / "$ means the units are any-one-optional in use.

${ }^{6}$ This discussion has been preserved in the previous part of this chapter.
} 
Possible World Theory is widely utilized in the analysis of ECC. Stalnaker (1968) proposed a possible-world semantics for counterfactual conditional. Digression on possible world semantics for modal logic: “ $\square p$ " (necessarily, p) is interpreted as " $p$ is true in all worlds which are accessible from the actual world." The accessibility relation $R$ between worlds can have various properties. If $R$ is transitive, then $\square p \rightarrow \square \square p$ is valid. If $R$ is symmetric, then $\biguplus p \rightarrow p$. If $R$ is serial, then $\biguplus \mathrm{p} \rightarrow \square \diamond \mathrm{p}$, where “ $\diamond \mathrm{p}$ " (possibly, p) is true if and only if “ $\square \square \neg \mathrm{p}$ ” is true.(pp. 121-123)

Stalnaker introduced a selection function $\mathrm{f}(\mathrm{p})=\mathrm{w}$ which maps each false sentence (or proposition) $\mathrm{p}$ into "the world $\mathrm{w}$ such that $\mathrm{w}$ is the minimal revision of the actual world that would be required to make $p$ true." Thus, we have the following account of the counterfactuals:

$$
\text { (S) } p \square \rightarrow q \text { if and only if } q \text { is true at } f(p) \text {. }
$$

Stalnaker's account has various virtues. It is able to match certain intuitions about non-truth-functional conditionals. Stalnaker's assumption that there is a unique closest $p$-world to the actual world turns out to be equivalent to the following pair:

$$
\begin{aligned}
& \text { (Conditional Excluded Middle): }(p \square \rightarrow q) \vee(p \square \rightarrow \neg q) \\
& \text { (Stalnaker's Axiom): If } \diamond p \text {, then } \neg(p \square \rightarrow q) \text { if } p \square \rightarrow \neg q
\end{aligned}
$$

David Lewis has pursued the counterfactual question to a totally new level, where many critical questions are found and released. David Lewis (1973a) took Stalnaker's account to task for his account of counterfactuals. In particular, Lewis objects to the assumption that there is a unique $f(p)$.

Lewis proposes the following variation on Stalnaker's approach:

(L) $p \square \rightarrow q$ if and only if some (accessible) $p \& q$-world is closer to the actual world than any $p \& \neg q$-world, if there are any accessible $\mathrm{p}$-worlds. If there are no accessible $p$-worlds, then $p \square \rightarrow q$ is true.

Lewis rejects two assumptions implicit in Stalnaker's account. 1, Uniqueness Assumption is the assumption that there is a unique closest $p$-world to the actual world. 2, Limit Assumption is as we proceed to closer and closer p-worlds, we eventually hit a limit and can go no farther.

Lewis and Stalnaker both accept the following two assumptions: Ordering Assumption is that the comparative similarity imposes a weak ordering connected and transitive on the accessible worlds, Centering Assumption is that the actual world is accessible from itself (indeed, they assume reflexivity of R), and closer to itself than any other world is to it.

Sweetser's $(1990,2002)$ classification of three domains - content, epistemic and speech act domains-explains the three types of the logic relations in conditionality. Dancygier (1987) retains the Sufficient Hypothesis. She claims that conditionality operates in the three domains, and that in each domain the Sufficient Hypothesis can be maintained. The following description briefly introduces this classification based on their research:

According to Dancygier (1987), the domains are linked through a metaphor which develops the meaning from the substantially physical domain to the highly-abstract social domains. That is to say, meaning is extended from concrete relations to subjective mental ones. In the content domain relations, causal relations hold between the described events and situations. (p.17) According to Sweetser (1990), the content domain is the source. In this level of conditional, the conditional "if-then" conjunction indicates that the realization of the event or state of affairs in the apodosis (p.114)

Sweetser (1990) asserts that in epistemic conditionals, the conditional "if-then" conjunction expresses the idea that the knowledge and the truth of the hypothetical premise expressed in the protasis would be a sufficient condition for concluding the truth of the proposition expressed in the apodosis. (p.116) In a word, the conditional relation is that between knowledge and a conclusion.

And according to Dancygier (1987) In speech act conditionals, " $p$ 's are used as comments on the speech acts performed in $q$ 's" (p.34)

Generally speaking, if an event causes another event, the relation is content conditional; if the knowledge of the event causes a conclusion, it is epistemic; if a speech act is performed, it is in the speech-act relation.

Epistemic stance is crucial in the cognitive analysis of conditional. Epistemic stance is the epistemic relationship which the speaker obtains of the world that is represented by the conditional sentence: the speaker might regard it as the actual world, might regard it as distinct from the actual world, Fillmore (1990) notes "or might not know whether the alternative world represented in the conditional sentences is the actual world or not". (p.142)

Fauconnier (1997) analyzed the epistemic stance, in terms of mental spaces and in English tense and mood. (p. 93)

Mental spaces are seen in Fauconnier (1994) as domains of "backstage cognition" (p.7), that is, the abstract mental constructs that are generally set up on the basis of general scenarios. What is "backstage cognition"? Fauconnier says,

Perhaps for the first time a genuine science of meaning construction and its dynamics has been launched. This has been achieved by intensively studying and modeling the cognition that lies behind the language and goes far beyond it, but which language reflects in certain ways, and which in turn supports the dynamics of language use, language change, and language organization. Echoing Erving Goffman, I have called this backstage cognition. (Fauconnier, 1994)

In this sense, mental spaces are actually mental constructs of potential realities which are created dynamically as a "conceptualizer" which is thought to be able to capture a string of speech when people hear a piece of language or a text 
when people read. "Examples of these are: the world defined by a picture, a world of fiction, the world of a person's beliefs and desires, time slices hypotheticals, or "umwelts.",7

Basically speaking, the theory of mental spaces can be understood as a theory defining referential structure. In this framework, Coulson (2001) meaning "depends on our ability to delimit the orbit of reference".(p.25) In the early stage, the theory was originally envisaged by Jackendoff (1975) and Nunberg (1978) respectively to deal with the intricate problems of indirect reference and referential opacity. Therefore the theory has thus been exploited to account for a lot of phenomena of different nature in language and thought. (See Fauconnier 1997 and Fauconnier \& Sweetser 1996 for a revision).Among these phenomena, what is most relevant to the present study is the considerations of conditionals and counterfactuals.

In mental space theory, language comprehension and production are considered in Fauconnier (1994), Sweetser and Fauconnier(1996), Dancygier and Sweetser(1996) as involving the setting-up of structured and interconnected cognitive domains and these domains are independent of language. Ferrari (2002) gives the statement of "Linguistic expressions are conceived as surface manifestations of these subjacent and highly abstract constructions" (p.221). The understanding of the use of language comes from the construction of a configuration of spaces hierarchically related and interconnected. During the course of the processing of each sentence in this piece of language, with the help of lexical and grammatical clues, the configuration of spaces is made dynamically active and therefore spaces are shaped into structures by the activation of frames and schematic conceptualizations. Lakoff (1987) names it as Idealized Cognitive Models. What's more, the processes concerning inference and reasoning help to shape the spaces into structures. As the use of the piece of language goes deeper, new spaces appear as a result of clues given by space-builders, grammatical markers, or pragmatic information.

As to space-builders, they can take various forms: prepositional phrases, connectives, clauses that require complements, and so on. After a general description of the theory of mental spaces, now it's the turn for the analysis of conditionals in light of the theory of mental spaces. In a mental space analysis of conditional constructions, a configuration of spaces will function as an informational frame with deductive potential. Fauconnier (1985) proposes that the entire if $p, q$ construction builds up a single space. Later he puts forward an important revision by developing a formal analysis for dealing with tense, and this proves to be a more refined set of tools for the description of the structural properties of conditional constructions. In the revised analysis system, function and behavior of tense in those constructions are more effectively dealt with, and the nature of space embeddings for subordinate clauses is more subtly described.

As to Sweetser (1996), she tends to treat conditional constructions as setting up two spaces, a space for the protasis and an embedded space for the apodosis. As to her, content level, epistemic and speech act conditionals have quite different conditional relationship and hence a different mental space embedding structure between the two spaces set up respectively for the apodosis and the protasis. She argues that the "normal" tense restrictions, the familiar prescriptive grammar rules apply only to content level conditionals, the non-application of "normal" tense restrictions in epistemic and speech act conditionals is a result of the looser link between the two spaces originally set up for the protasis the apodosis.

\section{CI FRAMEWORK}

CI is a theory of human reasoning and thought. It's a result of the studies of a wide range of scientific disciplines, such as philosophy, psychology, logic and linguistics. Its application is also wide-ranged in the above disciplines. Fauconnier and Turner (1998) points out that "conceptual integration or blending serving a variety of purposes is a general cognitive operation on a par with analogy, recursion, mental modeling, conceptual categorization, and framing”. As one of the most influential cognitive theories, CI is the further development of both the theory of metaphor and mental-space theory. According to CI, "conceptual integration is a fundamental cognitive ability taking place at all levels of perception, of understanding, and of memory." (Fauconnier, 1998). Conceptual blending is a kind of cognitive course when people carry on a conversation or an activity, especially a creative thought or activity. The operation of conceptual integration employs four mental spaces - two input spaces, one generic space and one blended space. The spaces are connected (mapping) to construct a network - conceptual integration network — to carry out the operation. In Fauconnier and Turner's theory, there are four types of the network: simplex, mirror, single-scope and double scope. Multiple blend networks are also suggested based on them.

1) Mental spaces are small conceptual packets constructed as we think and talk, for purposes of local understanding and action. Four spaces are suggested by Fauconnier. (1994, 1998), and Fauconnier and Turner (2002). In the typical CIN, four mental spaces work together-two input spaces (the inputs), one generic space and one blended space. The input spaces contain some elements where the source of the information happens. Another space is the generic space, the structure that is recognized as common of both input spaces is constitutes a generic space. The generic space is not structured by simple duplicating, but reflects some common and more abstract structure shared by the input spaces. The

\footnotetext{
${ }^{7}$ Methods and Generalizations. In T. Janssen and G. Redeker (eds.).Scope and Foundations of Cognitive Linguistics .Cognitive Linguistics Research Series. The Hague:Mouton De Gruyter.

${ }^{8}$ Shortened as CIN in this thesis
} 
blended space (the blend) includes the structure in the generic space, the specific structure in the inputs but not in the generic space and the structure even not in the inputs. Elements are combined and interact to produce new structure.

2) Cross-space mapping happens among spaces; mapping is the prerequisite part for blending. Figure 4.1 from Fauconnier and Turner (2002:46) shows these cross mapping relations:

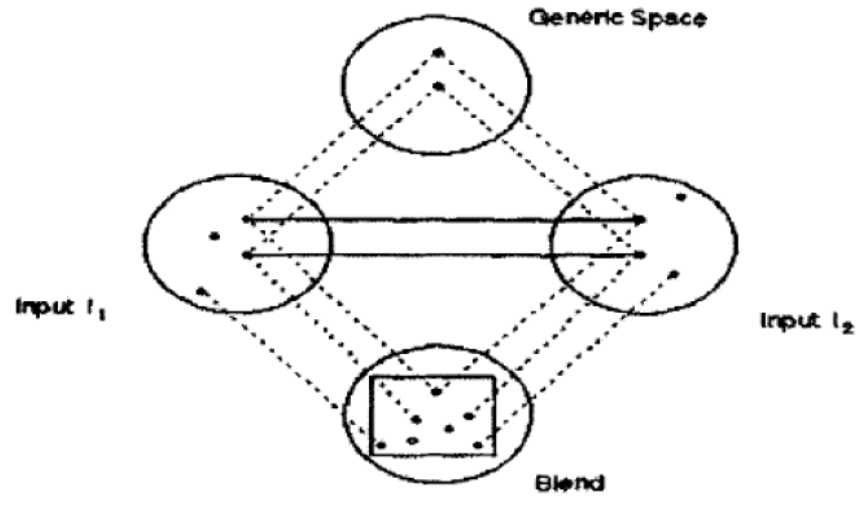

Figure 4.1 Cross-mapping

3) Fauconnier and Turner (1988) suggested that the blend has emergent structure not provided by the inputs and this emergent structure is crucial to the performance of the reasoning task. Composition, completion and elaboration lead to emergent structure in the blend. The blend has structure that is not from the inputs (Fauconnier and Turner, 2002).

Respectively, composition of elements from the inputs enables the relations available in the blend that do not exit in the separate inputs; completion brings additional structure to the blend; elaboration is an extended version of completion that results from imaginative mental simulation.

4) The inputs are not only the source of the projections to the blend but also receive reverse-projection from the developed blend. And, not all elements from the inputs are projected. That is to say, the projections from the inputs to the blend are the selective projection. Composition, completion and elaboration are used here, solely or together.

There are also principles for CI (all in Fauconnier \& Turner, 2002):

1) Access principle states that an expression that names or describes an element in one mental space can be used to access a counterpart of that element in another mental space. That is to say, if two objects (in the most general sense), a and $b$, are linked by a pragmatic function $F,(b=F(a))$, a description of a (the function expression), may be used to identify $b$.

2) The combination and interaction among elements and the way how the blending works is called constitutive principle.

3) Conceptual blending is subject to an array of competing optimality principles, or constraints for the process to be successful. The principles are in the following terms:

Integration: In the blend, scenarios are well integrated.

Topology: Elements in the blend should participate in the same kinds of relations as their counterparts in the inputs.

Web: The blend and the inputs are closely interrelated in implying the events.

Unpacking: The inputs and the network can be reconstructed for the blend.

Good Reason: Elements emerging in the blend should be meaningful.

These principles can be satisfied selectively, and the satisfaction of one may be contradictory to another, they are always satisfied to some extent in the on-line construction of the blend with the competition among the principles.

Network types are another important perspective:

Simplex network is a basic and simplest kind of CIN. One input has an abstract frame and another has the elements to fill in the frame. The cross-mapping is setting the value to the frame (or to be understood as a parameter in mathematics and logic). In the single-framing network, the two inputs consist of the elements essential for the integration. Usually the frame in one input is compatible with the elements in the other input and there are no clashes between the two inputs because only one frame is directly projected to the blended space to organize the blended structure. Fauconnier and Turner say (2002) "a simplex network does not look roughly like a blend at all, but it indeed is a perfect regular integration network, and is predictable from the theoretical principles of blending (p.120).

Mirror network is that when all spaces share an organizing frame. The common frame inheres in a richer frame provided by the blend. This type is a comparatively standard type of integration networks. In this network, all mental spaces, inputs, generic, and blend, share topology given by an organizing frame - a frame that specifies the features of the relevant activity, events and participants. An organizing frame provides a topology for the space it organizes, that is, it provides a set of organizing relations among the elements in the space. When two spaces share the same organizing frame, they share the corresponding topology and so can easily be put into correspondence. Establishing a cross-space mapping between inputs is straightforward when they share the same organizing frame. The input spaces mirror each other for all spaces in the network have the same organizing frame, so does the generic space. While spaces in a frame 
network share topology at the level of an organizing frame, they may differ at a more specific level. In the blend, some of the more specific features are present. The blended space also has that frame, in the blend, the common organizing frame of the network inherits in a richer frame that only the blend has. The elaborated frame only exists in the blend. Compression is quite frequent in the mirror network and compression of vital relations facilitates the run of the blend. In a mirror network, there are no clashes between the inputs at the level of an organizing frame, but there will be clashes at more specific levels below the frame level.

Single-scope network has two inputs with different organizing frames, one of which is projected to organize the blend. There are two separate organizing frames contained in the inputs, but only one of them is projected to the blend as its organizing frame. This kind network is the prototype of highly conventional source-target metaphor. The most obvious compression involves the use of pre-existing compression from the framing input. The projection of the source frame to the blend is linguistic construction used to evoke the source frame. Of course, there are projections from target input to the blend that also provide linguistic constructions for the blend, but they refer to elements below the frame level. Since the inputs have different frames, single-scope networks offer a highly visible type of conceptual clash.

Double-scope network differs from the single-scope frame, because its blend has an organizing frame taking parts from each frame from the both inputs. If the inputs are organized by different frames but some topology is projected from both frames to the blend, the network is also called the two-sided network. In this kind of network, both organizing frames make central contribution to the blend, and their sharp differences offer the possibility of clashes. Those clashes offer challenges to the imagination, and thus the blends can be highly creative. The inputs are not simply juxtaposed in the blend; rather, the emergent structure specific to the blend is created in the blend.

It's worth mentioning that these four types are not totally separated and independent from each other, the extension, cooperation of them empower our way of thinking.

Moreover, multiple blends are involved on a more absolute basis in our live thoughts. Fauconnier proposes a more general form of the network, called the multiple blends where a dynamic operation over any number of mental spaces that moreover can apply repeatedly, its outputs becoming inputs for further blending. In this more general scheme, it is still found that the defining features of conceptual integration: cross-space mappings between inputs, selective projection of generic spaces. But there are two main ways in which networks can be multiple blends: Either several inputs are projected in parallel, or they are projected successively into intermediate blends, which themselves serve as inputs to further blends.

Fauconnier $(1994,1998)$ insisted that conceptual blending exists in nearly all cognitive activities. The counterfactual reasoning and the construction of the unreal are studied by him and other scholars who utilize CI and relevant cognitive reflections. Fauconnier and Sweetser (1996) discussed some aspects of mental space theory and grammar, Dancygier \& Sweetser (2005) analyzed conditional constructions in "Mental Spaces in Grammar",

King, Keohane and Verba argue that there is no form of causal inference in the social sciences that does not depend upon counterfactual reasoning. Analyzing causality for social events is a matter of contrasting what in fact happened with counterfactual scenarios of what might have happened under different conditions.

(Fauconnier and Turner, 2002:218) According to this view, conceiving of a counterfactual scenario is a simple matter of making a change in the actual world and observing the consequences of that change.

However, Fauconnier and Turner (2002) holds that changing any one element opens up complicated questions of what else would need to be changed in order for that element to differ. Counterfactual reasoning is not a matter of imagining what we would have to change in the real world for the counterfactual scenario to be possible. (pp. 218-219)

Furthermore, counterfactual thought is also taken as causal logic. Extreme idea, as Roses and Olson believes "all counterfactual conditionals are causal assertions". Fauconnier and Turner (2002) argued against this extreme assertion with a "woman-in-coma" example, and exhibited it as logic of propriety. (p. 219)

Counterfactual reasoning is an everyday event that usually goes unremarked> Grammar can also use "plain" formswith no specific inflexions - to prompt counterfactuality. Varieties of structures are applied to counterfactual thought's manifestation.

Counterfactual spaces, as we commonly believe, are opposite to the "actual" spaces of. We have taken it or granted that counterfactuality is something imaginary, non-actual, not true or fictive.

In Fauconnier's research of CI, this is not right. He (2002) stated "counterfactuality is forced incompatibility between spaces, and when one is thinking about reality, counterfactuality is often a vital relation between spaces that involves some of the same people and same events (the elements in spaces - noted by the author)". (p.230)

He also suggested that counterfactual thought happens in any case, when we mark the spaces as "actual", counterfactuality in counterpart spaces is also involved. As the book's title states, counterfactuality is "the way we think".

Language is thought by the cognitive linguists to provide information that triggers reasoning process. For example, he says (1997) "premises for a deductive argument or the specification of a structure that will be used analogically". (p. 99)

As is said (1997), he defines language as an active involvement of "setting up construals, mappings between domains, and discourse configurations, with the fundamental properties of Accessing, Spreading, and Viewpoint. (p.99) 
Explicitly, expressions of natural language are only the most economical manifestation that activates complex mental projections among domains. The important mechanism based on which these projections can take place is analogical counterfactual reasoning.

He (1997) asserts "the analysis of counterfactual conditionals is no fussy little grammatical exercise. If it is lack of the means for interpreting counterfactual conditionals, we can hardly claim to have any adequate philosophy of science." (p. 99) Meanwhile, analogy enables the mappings among spaces and logic connectors to happen between counterparts in different spaces.

Fauconnier and Turner (2002 once set "Watergate in France" (p. 225) as an example as follows:

"In France, Watergate would not have hurt Nixon." This counterfactual can have many readings, but a typical one contrasts the American and French cultural and political systems. It brings in aspects of the French system from one input and the Watergate scandal and President Nixon from the other. In the blend, we have a Watergate-link situation in France, but running the blend delivers attitudes quite different from those in the American input.

A CIN is set up to explain this counterfactual reasoning:

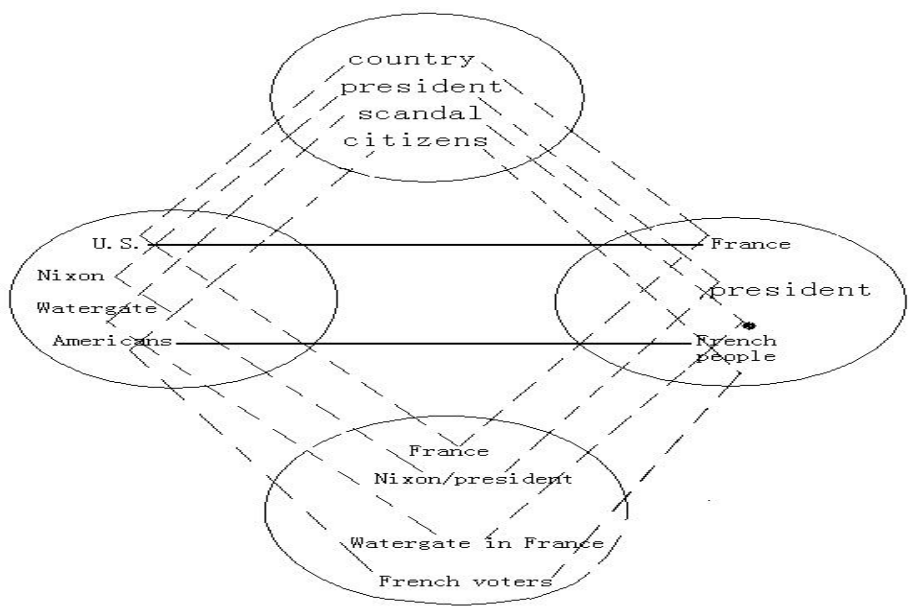

Figure 4.2 Nixon-France Network ${ }^{9}$

A counterfactual frame is stated:

1) country has president/leader elected by citizens

2) president is head of political party competing with others for leadership of country

3) president's actions are constrained by laws, public reaction...

4) action brings harm to president if

--it triggers negative public reaction

--it is unlawful and president is punished, etc

The epistemic is involved in the reasoning-world knowledge about Watergate, Richard Nixon, break-in, tapes, lies, impeachment and resignation.

The running of the blend is in the emergent structure: French Watergate scandal doesn't harm French president Nixon. Analogy and disanalogy are between the counterparts in the spaces.

Therefore, the real conditional goes as the follows:

1) X: "If the price increases, I won't buy the car."

(Tthere is a man who plans to buy a car. He has a certain sum of money for the purchase, and the target merchandise has been appreciated. Upon the purchase, the car dealer calls to inform him a possible recent increase of the price, the man replies like above)

Here, the condition of a more expensive price is thought to be "real", so this sort of conditional is called real conditional. To analyze the meaning of this conditional, we can set up a CIN along the following steps:

1) There the elements in the two input spaces, with counterparts analogized with each other. ("Not buying" vs. "Buying", "More expensive price" vs. "Maintained price", the Buyer and the Merchandise), and cross-space mapping take place between the two spaces, as in Figure 4.3 below:

\footnotetext{
${ }^{9}$ Drawn by the author with a slight change in shape from the original in Fauconnier and Turner, 2002:226; and the tables and figures in this thesis, if not specified, are drawn by the author.
} 


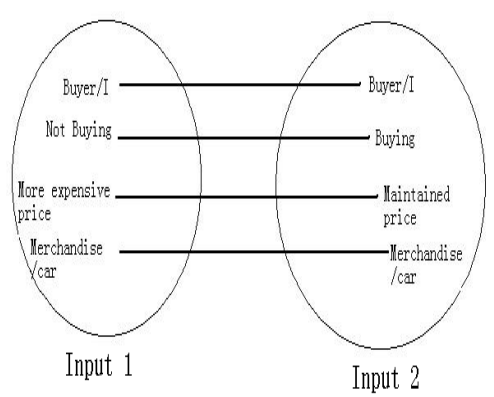

Figure 4.3 Input spaces of car-purchase $\mathrm{X}$

In input 1, there are the following elements: a buyer (I), an action (not buying the car), a price (more expensive) and a merchandise (the car); in input 2, the buyer and the car still in their existence while the action is "buying" instead, and the price is a "maintained price".

2) In the generic space, the elements are in abstraction, namely "Buyer", "Action", "Price" and "Merchandise", as in Figure 4.4

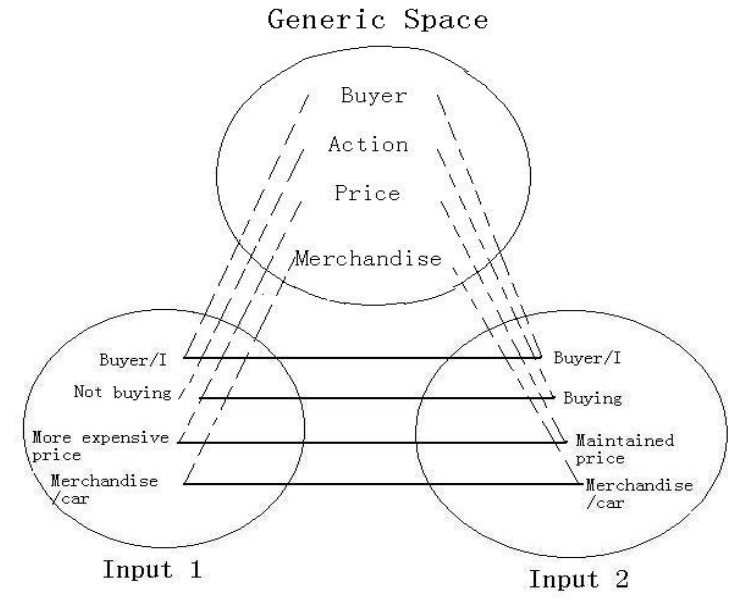

Figure 4.4 Generic space of car-purchase X

3) The frame from the Inputs are projected to the blend, and the blend space is yielding the meaning, the CIN is completed for the reasoning, as in Figure 4.5:

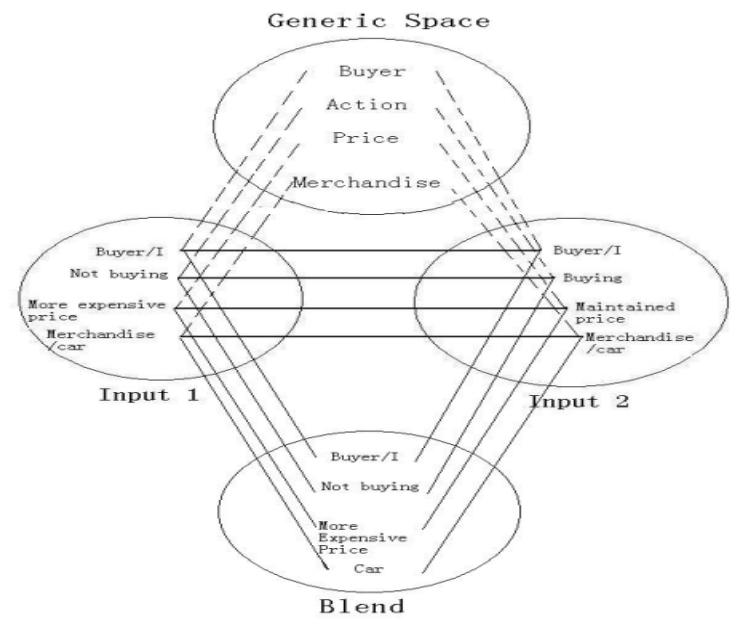

Figure 4.5 CIN of car-purchase X

It is easy to find that projected to the blend are those elements in Input 1, but not those in Input 2. According to the types of networks that we have reviewed in the above chapter, it is obvious that this CIN is a simplex type of CIN. The frame projected to the blend is selected only from one of the inputs.

A question is asked: Why "I won't buy the car" from Input 1 is selected. The way how the composition/completion/elaboration (see 2.3.4.5) to do must be decided. 
Carefully contrasting Input 1 and Input 2 in the car-purchase case, we find that there are relations among the elements, not only through the cross-mapping among the counterparts in each input, but also a certain kind of relation connecting the elements respectively in each input. This relation is what we understand as the frame in the conceptual integration. In this case, the element "more expensive price" is connected with "not buying" to construct a condition frame. A causal relation exists in this frame of two elements.

Obviously between "maintained price" and "buying" exists the same relation, but only the frame in Input 1 is projected to the blend. Observing the case again, it is clear that the frame in Input 1- "more expensive price $\rightarrow$ not buying"-is explicitly manifested in the language structure - the statement $X$. However, the frame of "maintained price $\rightarrow$ buying" is an implied condition. It seems that only the explicitly-manifested frame is operated in the blend as to convey the meaning. The process of the projection is shown:

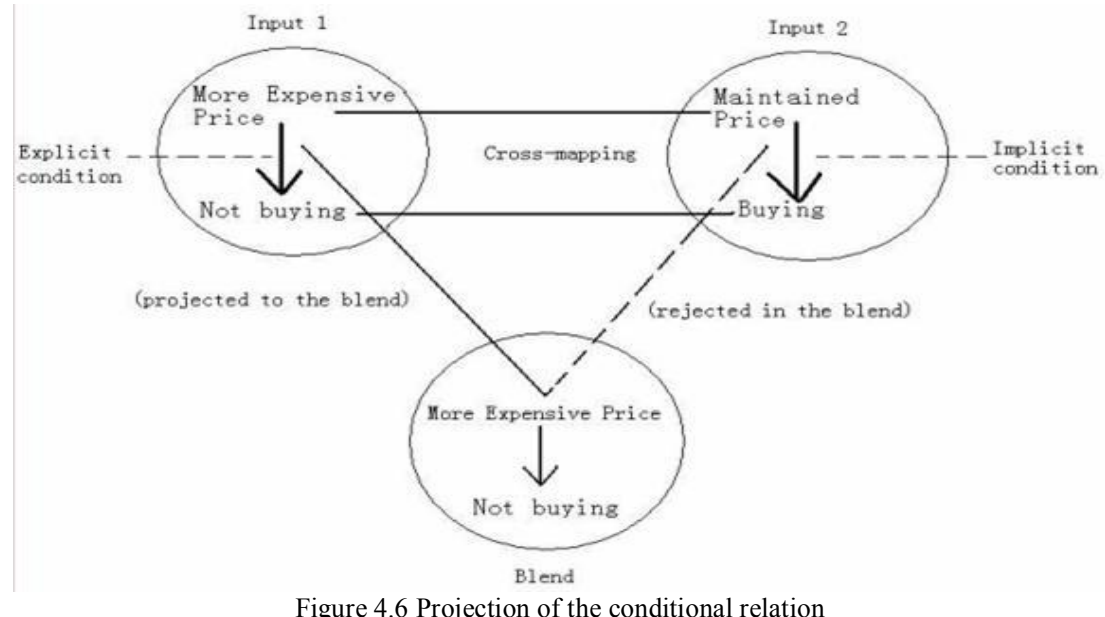

This selective projection is a matter of "completion", and the selective projection works here. Through the operation of completion, the explicit conditional relation is operated in the blend. Now, we have defined the operational principle in real conditional.

With the study of real conditional, ECC can be analyzed on a more absolute basis. We still use the steps in the previous verse to construct a network, to find the frame and the projection, then to define the principle according to which the counterfactual conditional CIN is to indicate the meaning.

Let's look at another example

2) Y: If the price of the car increased, I wouldn't buy it.

Statements $Y$ is a typical counterfactual conditional based on the pedagogical grammar rules. Specific verb forms are used to indicate that this sentence is of the subjunctive mood. This sentence is a good enough sample for the following analysis.

Now the question is how this ECC conveys the counterfactual meaning. the CIN shown in Figure 3.6 is employ to prepare for further analysis. The blend in the network has an emergent structure which carries a negative meaning that the man will not buy the car. In a word, the action is negative.

However, the meaning of the statement $Y$ is: 1) the man will buy the car; 2) the increase of the price is believed not to happen. So the network must be re-organized as is shown in Figure 4.7:

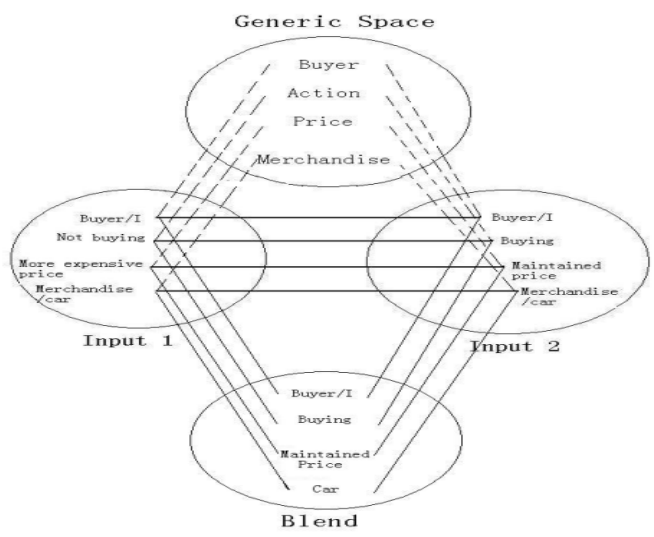

Figure 4.7 CIN of car-purchase $Y$

This time, we choose the implicit conditional relation in Input 2 for the reasoning. If we analyze it, we find that the epistemic stance plays an important role here. Because the man is reassured by the car dealer that the price won't 
increase, in his epistemic stance the condition "more expensive price" and the consequence "not buying" won't really happen. This is the key of the counterfactual conditional blends.

Furthermore, in the counterfactual conditionals that have specific verb forms, the grammatical features add information to the epistemic stance that we would take into consideration in our reasoning. If the conditionals have no specific forms - the implied counterfactuality--phonological property of the utterance, the pragmatic property, context, and other non-linguistic factors such as culture, psychological status etc. are taken into the epistemic consideration, too.

$\mathrm{CI}$ has powerful explanatory capability in reasoning including counterfactual reasoning. Fauconnier and Turner (2002: 150) declared "composition, completion and elaboration lead to emergent structure in the blend. The blend has structure that is not from the inputs..."

Classic CI only enables a "black-box" operation in producing the emergent structure. This thesis attempts to provide a clearer description of the operations in the counterfactual conditional semantics.

Some basics and crucial notions of the blending theory, its researches and the general description of the theory are reviewed in this chapter, with the ambition of sorting out a basic knowledge of CI. After the introduction and elaboration in this chapter, we may have gained some fundamental knowledge about the conceptual integration theory as the necessary preparation for the further analysis of the roles that CI plays in interpreting the counterfactual conditional meanings.

Conceptual integration is in fact a fundamental aspect of all human experience. Fauconnier and Turner claim that integration is involved in everything from perceptual processing, to the experience of physical senses to the knowledge of logic and reasoning.

Many scholarly approaches have studied a large amount of human daily phenomena through CI. As we have discussed in this chapter, conceptual blending is a universal process and is obvious at many levels of analysis, including the conceptual structure of some concepts, grammatical structure in sentences, an overarching level across conceptual domains and meaning construction of gesture. Fauconnier \& Turner (1995) explored the multiple-blends by some new examples and showed that formal expression in language is also a way of prompting hearer and reader to assemble and develop conceptual construction and blending. Sweetser (2002) analyzes the blending on performative speeche and action. Coulson (2001) develops conceptual integration of rhetorical strategies, noun and adjective compounding. Turner (1996), Oakley (1995), Freeman (1997) apply elaborate analysis of mental space construction and integration in literary narratives, poetry and general rhetoric. Zbikowski (1997) shows how conceptual integration can apply to music.

As the above study indicates, CI can be used to address both the linguistic and non-linguistic domains. It is powerful to enunciate many kinds of language forms and phenomena. It is universal and formidable in human cognition. Some examples have been quoted in this chapter to show the detail process how CI applies in the daily human thoughts, which is reserved for the following analysis on its explanation to ECC.

\section{MAJOR FINDINGS AND SUGGESTIONS}

The pattern of constructing counterfactual conditional CIN can be drawn as a principle now:

In CIN of counterfactual conditionals, the implicit conditional frame not in the language is projected to the blended space, because it is valid in epistemic stance. With the contrast in CIN of non-counterfactual conditionals, the explicit conditional frame in the linguistic expressions is projected to the blended space.

It's worth mentioning that there are fictive elements in the counterfactual inputs. They are fictively analogical to the counterparts. Fauconnier and Turner's description of counterfactuality (see 2.3.4.2) helps to explain this. Counterfactual thought happens in any case. Though sometimes we mark the spaces as "actual", we also involve counterfactuality in counterpart spaces. This can explain the occurrence of the fictive elements and the fictive logic.

We can utilize a function logic $y=F(x)$ to describe counterfactual conditional CIN. We can change variable " $x$ " into " $r$ " to indicate the explicit conditional relation and " $r$ " for the implicit conditional relation, variable " $y$ " is changed into " $p$ " for the projection to the blend, and the computer $F$ is changed into " $I$ " for the CI process. Since epistemic stance is involved, we add a dynamic computer " $e$ " standing for the epistemic stance attached to "I". So, the principle is formalized into the functions:

In CIN of counterfactual conditionals, $p=I e(-r)$; with the contrast in CIN of non-counterfactual conditionals, $p=I e(r)$. In these formula, the standard of the projection in the selection of the relations are symbolized and generalized by the two parameters--(-r) and (r); the epistemic stance is indicated by the computer " $e$ " and these two aspects of conceptual integration make the cognitive processing in the blends about counterfactual conditional reasoning apparent, but not a "black-box".

This thesis discusses how the counterfactual conditional meaning is conveyed from a cognitive perspective and a few examples are analyzed on the basis of a pattern developed from the CI. The analysis is a gradual course since the reasoning is moved from plain conditional logic to counterfactual logic.

Conceptual integration is a basic mental capacity that leads to new meanings, global insight, conceptual compressions useful for memory and manipulation of otherwise diffuse ranges of meanings. It plays a fundamental role in the construction of meaning in everyday life, in the arts and sciences, in technological development, and in religious thinking. The essence of the operation is to construct a partial match between input mental spaces and to project 
selectively from those inputs into a novel blended space, which the dynamically develops emergent structure (Fauconnier 2001: 1). Up to now, it has provided a good explanation in many linguistic and non-linguistic fields.

With the development of cognitive researches on language itself, we find that CI could have possibilities for exploring the hidden mechanism involved in the interpretation of ECC. Conceptual integration has complete networks, which function independently according to set of uniform structural and dynamic principles. The basic operation compromises four mental spaces: two inputs, on generic space and one blended space. In blending, structures from inputs are selectively projected to a separate blended space. Through composition, completion and elaboration, the blend develops structures that are not provided by the inputs.

We suggest a highly formalized framework according to which the projection course is clearly described. Then we analyze some examples to complete the justification of the framework.

In the analysis, CI is utilized as the general principle to explain how meaning is generated and understood. In the use of the framework, variables are valued according to specific ECC semantic elements, specific verb forms and structures. Different kinds of ECCs collected from pedagogical grammar book can all be well explained.

According to the framework, a pair of formula is established form the explanation of the non-counterfactual conditionals to that of ECC.

There are also suggestions for the future research:

Firstly, some limitations inevitably arise due to our limited time and energy. With regard to ECC, its countless studies and theories can not be exhausted by this thesis, as being a brief initial study to the rather complicated phenomenon and its more complex and numerous theories.

Secondly, CI theory is rather a general psychological theory which covers a wide range of mental reasoning phenomena. In the application of it and even intending to further it on such a specific matter like ECC, possible misunderstanding and misuse of it might happen in the description and analysis.

Finally, that the copra is open may also mean that the non-exhausted data can't cover every possibility of ECC, which might lead to a not perfect enough complete conclusion of the research.

We will be very pleased if more studies will be carried on this issue. That CI theory will be profoundly utilized in the further researches to explain away the mist in linguistic issues including the meaning conveyance of ECC is also expected by any research like the author myself.

\section{REFERENCES}

[1] Asher, E.A. (1994). Conditionality. In The Encyclopedia of Language and Linguistics. Oxford: Pergamon Press.

[2] Bennett, J. (2003). A philosophical guide to conditionals. Oxford: Oxford University Press.

[3] Bolinger, D. (1997). Meaning and Form. London: Longman.

[4] Chalker, W. (1984). Current English Grammar. London and Basingstoke: McMillan Publishers Ltd.

[5] Celce-Murcia \& Larsen Freeman. (1999). The Grammar Book: An ESL/EFL Teacher's Course. Boston, MA: Heinle and Heinle Publishing Company.

[6] Dancygier, B. \& E, Sweetser. (1997). Then in conditional constructions. (pp.109-36) In Cognitive Linguistics 8.

[7] Dancygier, B. (1998). Conditionals and Prediction. Cambridge: Cambridge University Press.

[8] Dancygier, B. \& E. Sweetser. (1996). Conditionals, distancing and alternative spaces. (pp.83-89). In A.E. Goldberg (eds.). Conceptual Structure, Discouse and Language. California: CSLI Publications.

[9] Dancygier, B. (1998). Conditionals and Prediction: Time Knowledge, and causation in conditional construction. Cambridge: Cambridge University Press.

[10] Eckersley, C.E. \& J. M. ckersley. (1960). A Comprehensive English Grammar for Foreign Students. London: Longman

[11] Eckersley, C.E. \& Eckersley, J. M. (1960). A Comprehensive English Grammar: For foreign students. London: Longman Group Ltd.

[12] Fauconnier, G. (1994). Mental Spaces. Cambridge: Cambridge University Press.

[13] Fauconnier, G. (1997). Mappings in Though and Language. Cambridge: Cambridge University Press.

[14] Fauconnier, G. \& M. Turner. (2002). The Way We Think. Basic Books.

[15] Fillmore, C.J. (1990,a). Epistemic stance and grammatical form in English conditional sentences. (pp.137-162). In M.Ziolkowski, M. Noske \& K. Deaton (eds.). Papers from the $26^{\text {th }}$ Regional Meeting of the Chicago Linguistic Society. Chicago: Chicago Linguistic Society.

[16] Fillmore, C.J. (1990,b ). The contribution of linguistics to language understanding. (pp.109-128). In A. Bocaz (eds.). Proceedings of the $1^{\text {st }}$ Symposium on cognition, Language and Cultre. Universidad de Chile.

[17] Goodman, N. (1947). The problem of counterfactual conditionals. (pp.113-120). In Journal of Philosophy, 44.

[18] Lakoff, G. and Johnson, M. (1999). Philosophy in the Flesh: The Embodied Mind and Its Challenge to Western Thought. NY: Basic Books.

[19] Leech, G. (1983). Principles of Pragmatics. London: Longman.

[20] Lewis, D.K. (1973). Counterfactuals. Oxford: Basil Blackwell.

[21] McLaughlin, R.N. (1990). On the Logic of Ordinary Conditionals. Albany: State University of New York Press.

[22] Nayef, K. \& A. Hajjaj. (1997). Errors in English among Arabic Speakers: Analysis and Remedy. Beirut, London: York Press and Librairie du Liban.

[23] Palmer, F.R. (1974). The English Verb. London: Longman.

[24] Quirk et al. (1985). A Comprehensive Grammar of the English Language. London: Longman. 
[25] Sweetser, E. (1990). From Etymology to Pragmatics: Metaphorical and Cultural Aspects of Semantic Structure. Cambridge: Cambridge University Press.

[26] Sweetser, E. \& G, Fauconnier. (1996). Cognitive links and domains: basic aspects of mental space theory. (pp. 1-28) In G. Fauconnier \& Sweetser (eds.). Spaces, Worlds and Grammar. Chicago and London: The University of Chicago Press.

[27] Van Dijk, T.A. (1979). Pragmatic connectives. (pp.447-456). In Journal of Pragmatics: 3.

[28] Bo Bing \& Zhao Dexin. (1978). A Handbook of English Grammar. Beijing: Commercial Press.

[29] Liao Qiaoyun. (2005). C-R-AModel: A Full Dimension Explanation on Language Communications. Chengdu: Sichuan University Press.

[30] Lu Gusun et al. (2001). A Grand English-Chinese Dictionary. Shanghai: Shanghai Translation Press.

[31] Shi Yuzhi. (2000). The Cognitive Basis of English Grammar. Nanchang: Jiangxi educational Press.

[32] $\mathrm{Xu}$ Lijie. (2005). Conditionals and Subjectification: A Cognitive Approach to the Study of English IF-Conditional Constructions. Shanghai International Studies University PhD Thesis.

[33] Wang Yin. (2001). Semantic Theory and Language Teaching. Shanghai: Shanghai Foreign Language Teaching Press.

[34] Wang Yin. (2006). Cognitive Linguistics. Shanghai: Shanghai Foreign Language Teaching Press.

[35] Zhang Daozhen. (2002). A Practical English Grammar. Beijing: Foreign Language Teaching and Research Press.

[36] Zhang Zenbang. (1984). A New Edition of Brief English Grammar. Shanghai: Shanghai Foreign Language Teaching Press.

[37] Zhao Yanfang. (2000). A Brief Introduction to Cognitive Linguistics. Shanghai: Shanghai Foreign Language Teaching Press.

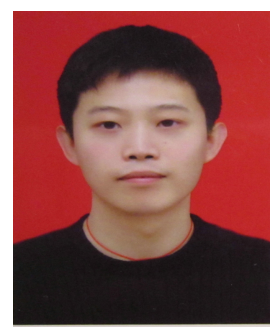

Ling Qin, born in Ya'an City, Sichuan Province, China. April 13, 1979. Graduating from Sichuan International Studies University (SISU), Chongqing City, China. Acquiring the Master Degree of Literature in 2009. Now Majoring in Linguistics.

He works in Sichuan Agriculture University (SAU) now as a Lecturer and used to be a part-time teacher in SISU. He has published 2 essays. Namely:

Qin Ling (2008). Xun Zi's 'Name' and 'Fact' Notions and the Semantic Causal Theory. (p.176). Journal of Sichuan International Studies University. 